REVIEW ARTICLE

\title{
Allergic Reaction due to Anti-Tuberculosis Drugs, How to Manage?
}

\author{
Nur Prasetyo Nugroho*, Tutik Kusmiati \\ Department of Pulmonology and Respiratory Medicine, Faculty of Medicine Universitas Airlangga, Surabaya, \\ Indonesia.
}

\section{ARTICLE INFO}

Article history:

Received 05 July 2020

Received in revised form 05 May

2021

Accepted 19 May 2021

Available online 31 May 2021

\section{Keywords:}

Tuberculosis,

Allergy,

Drug challenge,

Desensitization.

\begin{abstract}
Tuberculosis (TB) still becomes a significant health problem in Indonesia. The first-line anti-tuberculosis drug (ATD) is still the most effective TB drug, but it can have some side effects. One of them is allergic skin reactions that can affect a patient's compliance. Allergic reactions due to ATD are found in 4-6\% of TB cases and are the third most frequent side effect after gastrointestinal and liver function disorder. All first-line ATD can cause allergic reactions. Allergic reactions due to ATD can be mild, such as itching and reddish rashes, to severe and life-threatening rashes, such as anaphylactic shock, Steven Johnson Syndrome (SJS), and Toxic Epidermal Necrotic (TEN). The most important things in the management are identifying and stopping drugs. It includes drug challenge and desensitization of causing drug. Desensitization must be distinguished from drug challenge or provocation tests, which are diagnostic tools. The proper management of ATD allergic reactions can improve compliance and patient's outcomes.
\end{abstract}

\section{INTRODUCTION}

Tuberculosis (TB) is an infectious disease caused by Mycobacterium tuberculosis. It is still a significant health problem in the world. ${ }^{1}$ Every year, it is estimated that there are 8 million new cases and 2 million deaths due to TB. ${ }^{2}$ The recommended first-line antituberculosis drugs (ATD) is a combination of Isoniazid, Rifampicin, Ethambutol, and Pyrazinamide for six months. First-line ATD has been proven effective in killing TB germs, although it can cause side effects, including liver dysfunction (1.74-13.9\%), gastrointestinal disorders (1.64-10\%), and skin allergies $(1.43-7.5 \%))^{2,3,4}$

Allergic reactions due to ATD are found in 4-6\% of TB cases, which is one of the most common side effects. ${ }^{5,6,7}$ In the study of Aligazhar, et al. (2018), allergic reaction of ATD was the third most side effect after gastrointestinal disturbances and liver function. ${ }^{4}$ The studies of Marra, et al. (2007) and Amit, et al. (2017) also show the same results. ${ }^{2,3}$
All first-line ATD can cause allergic reactions. ${ }^{8}$ Allergic reactions due to ATD can be mild, such as itching and reddish rashes, to severe and life-threatening rashes, such as anaphylactic shock, Steven Johnson Syndrome (SJS) and Toxic Epidermal Necrotic (TEN). ${ }^{9}$ Allergic reactions due to ATD must be managed because they can cause treatment interruption.

\section{ANTI-TUBERCULOSIS DRUG ALLERGY Definition}

According to the American Academy of Allergy, Asthma, and Immunology (AAAAI), drug allergy is an immunological response caused by exposure to a drug insensitive or sensitive people. ${ }^{10,11}$ Drug hypersensitivity reactions are all side effects that are drug allergies. ${ }^{12}$ Drug hypersensitivity reactions consist of 2 types, namely allergic and non-allergic types. Drug hypersensitivity reactions involving the immune system are called drug allergies. ${ }^{13}$

*Correspondence: mazpraz.keren@gmail.com 


\section{Risk Factor}

Risk factors for drug allergies can be related to patient factors or drug factors. Patient risk factors include women, accompanying diseases (HIV, kidney disease, liver disease), ethnicity, polypharmacy, alcoholics, and genetics. Drug risk factors include the nature of the drug as a hapten, pro hapten, or the drug's ability to bind to immune receptors. The mode of administration also affects risk; topical, intramuscular, and intravenous administration more often causes allergies than oral; this is due to the presence of antigen in the skin and high drug concentrations achieved quickly with intravenous administration compared with oral administration. ${ }^{10,14}$

\section{Epidemiology}

Allergic reactions or drug hypersensitivity account for $15 \%$ of adverse drug events and affect $7 \%$ of the general population. ${ }^{13}$ Allergy to first-line ATD is one of the side effects in $5.7 \%$ of TB patients. It ranks the third most side effects of ATD after impaired liver function and gastrointestinal disorders. ${ }^{2,3,4,15}$ All firstline ATDs can cause rashes. This side effect in TB treatment ranges from $4.7 \%$ to $23 \% .^{8}$ The incidence of first-line ATD allergic reaction is $2.38 \%$ in pyrazinamide; $1.45 \%$ in streptomycin; $1.44 \%$ in ethambutol; $1.23 \%$ in rifampicin; and $0.98 \%$ in isoniazid. $^{15}$

The onset of rash usually appears in the first two months of TB treatment. ${ }^{8}$ The rash ranges from mild, such as maculopapular rash, to life-threatening ones, such as Steven Johnson Syndrome (SJS), Toxic Epidermal Necrolysis (TEN), and Drug Rash with Eosinophilia and Systemic Symptoms (DRESS). Maculopapular rashes or morbilliform are the most common in $95 \%$ of cases. ${ }^{8}$

\section{Pathophysiology}

The pathophysiology of drug allergy is not fully understood. However, according to Abe, et al., there are three hypotheses of cell-mediated immunity reaction and $\mathrm{T}$ cell activation in drug allergies ${ }^{15}$, namely:

- Hapten/pro-hapten hypothesis

- Interactions with immune receptors hypothesis $(p-i$ model)

- Altered peptide hypothesis

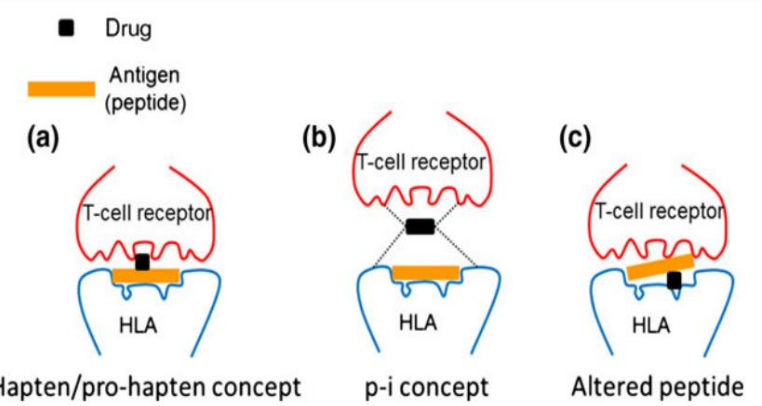

Figure 1. Drug interaction with immune system ${ }^{15}$

The first hypothesis refers to the hapten/prohapten hypothesis. The drug is not antigenic before it binds to protein. ${ }^{15}$ Drug molecules that enter the body act as hapten (small covalent molecules that bind to amino acids and proteins). Pro-hapten is a molecule that can turn into hapten after metabolism. Hapten and prohapten can act as allergens, antigens, immunogens, or sensitogens. They can cause hypersensitivity reactions in humans (Figure 1). ${ }^{10}$ Drugs that act as hapten bind covalently to serum proteins, including the Major Histocompatibility Complex (MHC) molecule. Bonding with $\mathrm{MHC}$ will activate $\mathrm{T}$ cells to initiate an immune response that causes systemic to skin reactions, and side effects can occur immediately or delayed. ${ }^{10}$

The second hypothesis, the p-i model introduced by Pichler, is the direct bonding of drugs with $\mathrm{T}$ cell receptors or Human Leukocyte Antigen (HLA). It can cause $\mathrm{T}$ cell activation and the occurrence of an immune response. ${ }^{10}$ The drug can bind to $\mathrm{T}$ and HLA cells without forming hapten. The changes of the previous paradigm state that drug induction in immunological reactions depends on the reactivity of the drug to the protein. Drug molecules that do not bind to proteins can stimulate $\mathrm{T}$ cells by binding to $\mathrm{MHC}$ and $\mathrm{T}$-cell receptors (TCR). ${ }^{15}$

The third hypothesis is the modified peptide. Peptides are protein fragments released by Major Histocompatibility Complex I (MHC I) in an immune response to activating $\mathrm{T}$ lymphocyte cells. In this hypothesis, it is stated that a drug molecule with a low weight can bind non-covalently to the HLA molecule through an antigen-binding gap, therefore changes occur in peptide repertoire. ${ }^{10}$ Because the drug binds directly to HLA, the HLA receptor gap will change. Peptides that bind to HLA will occupy holes with new shapes. If the subject is intolerant of unique peptides, T cells will 
be activated through interactions with MHC. ${ }^{15}$ In this process, there is no need for sensitization because the stimulation is directed at memory T cells and effectors. ${ }^{10}$

The most frequent ATD hypersensitivity reaction type is type 1 or immediate type. It occurs in $3.4 \%$ of TB patients who take ATD. The severity of ATD is usually grade 1 , but it can occur to grade $3 .^{14}$ Type 1 hypersensitivity reactions in TB patients are related to IgE levels. ${ }^{16}$ Total specific IgE levels in TB patients is known to be higher than in healthy people. It can be related to $M$. tuberculosis infection, which stimulates the work of T-helper 2 (Th2) to produce interleukin-4 (IL4), which stimulates B cells to produce IgE. ${ }^{17}$ Allergic desensitization is an essential treatment for type 1 hypersensitivity reactions. ${ }^{18}$ Clinical symptoms of each type of hypersensitivity reaction can be seen in Table 1 .

\section{Clinical symptoms and severity}

Clinically drug allergies are divided into immediate or delayed, depend on the onset after drug administration (Figure 2). The immediate type appears 1-6 hours after the drug is given (most often in the first hour). The symptoms include local erythema, urticaria, angioedema, conjunctivitis, rhinitis, bronchospasm, gastrointestinal disorders, anaphylaxis, or anaphylactic shock. Immediate drug hypersensitivity reactions may be mediated by $\operatorname{IgE}$ (type 1 hypersensitivity reactions). ${ }^{13}$ Non-immediate or delayed-type hypersensitivity reactions usually appear after the first 6 hours of drug administration. Common symptoms include maculopapular exanthema and slow type urticaria; often associated with a slow-type T-cell dependent allergic mechanism (type IV hypersensitivity reaction). ${ }^{13}$

ATD allergy symptoms include morbilliform rash, erythema multiforme, urticaria, lichenoid eruption, exfoliative dermatitis, and SJS. ${ }^{19}$ In a study of 9,111 hospitalized patients, 25 patients $(0.27 \%)$ experienced SJS with the leading cause of Thiacetazone. Pyazinamide ATD is the most common cause of allergy (2.38\%), followed by Streptomycin $1.45 \%$, Ethambutol $1.44 \%$, Rifampicin $1.23 \%$, and Isoniazid $0.98 \% .{ }^{20}$ There is no grading on the severity of allergic skin reactions due to ATD in general. The degree of severity is usually assessed from several types of skin allergic reactions due to drugs. SJS, TEN, DHS, vasculitis, FDE are assessed as severe allergic reactions. The need for hospitalization assesses the severity of the allergic reaction. ${ }^{19}$ Nowadays, the recommended grade of severity of ATD allergy is from the International Union of Tuberculosis Lung Disease (IUATLD) (Table 1). The various types of ATD allergic reaction can be seen in Figure 3. ${ }^{19}$

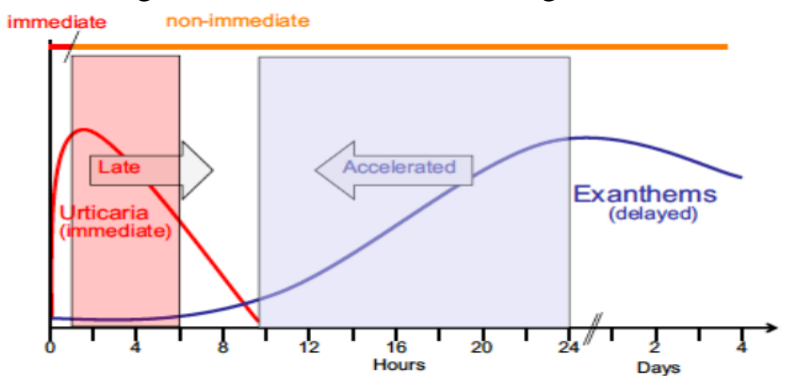

Figure 2. Distribution of drug hypersensitivity reactions based on onset 13
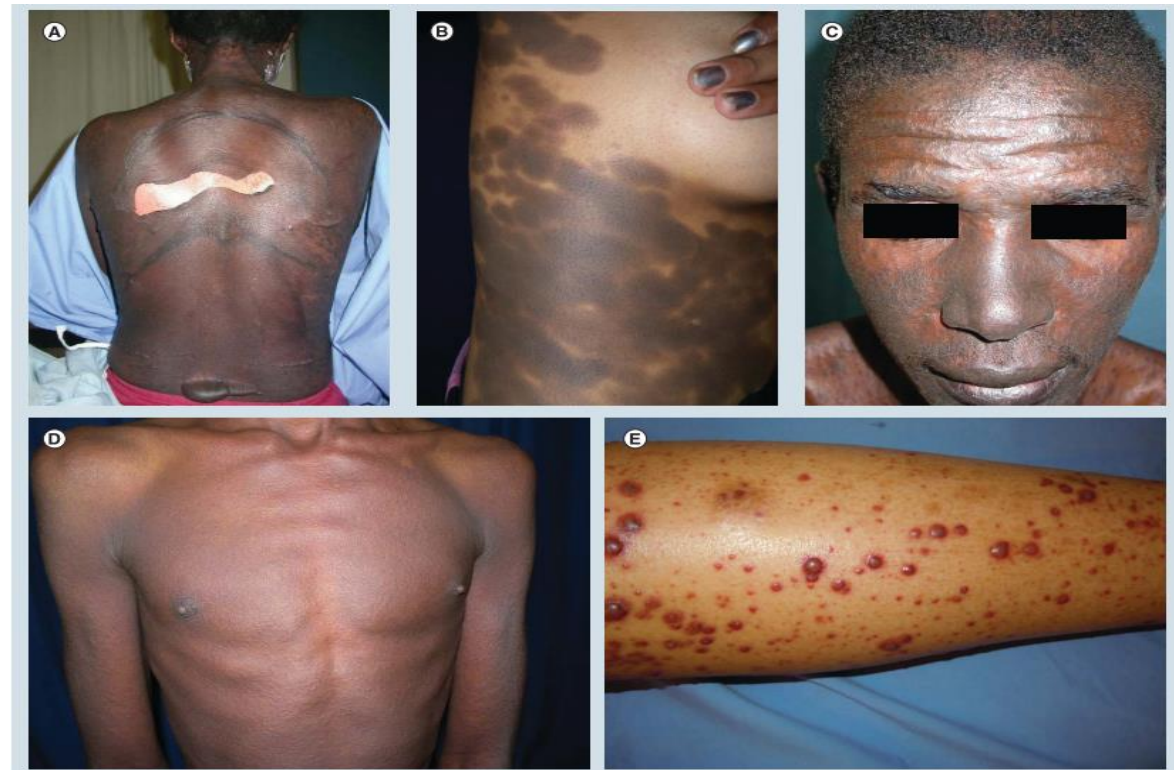

Figure 3. ATD Allergic reaction; A. Toxic epidermal necrosis with Nikolsky sign, B. Hyperpigmented lesions in fixed drug eruption, C. Purplish plaque on lichenoid drug eruption, D. Purpura in vasculitis due to drugs, E. Erythema morbilliform lesions. ${ }^{19}$ 
Table 1. The degree of severity of allergic skin reactions in ATD 20

\begin{tabular}{ll}
\hline Severity & Clinical symptomps \\
\hline $1^{\text {ST }}$ degree & Moderate itching or reddish rash \\
$2^{\text {nd }}$ degree & Extensive maculopapular rash with or without itching \\
$3^{\text {rd }}$ degree & Papular, vesicular, or wet rashes \\
& Purpura \\
& Skin or mucosal ulcer \\
$4^{\text {th }}$ degree & Bullous lesions (Lyell or Steven Johnson syndrome) \\
& Febrile erythroderma \\
& Skin necrosis (TEN) \\
\hline
\end{tabular}

\section{MANAGEMENT}

All ATD can cause allergic reactions. Assessment of the severity of allergies is very important for further management, including treatment interruptions and referral to more complete health facilities. $^{8}$

\section{ATD allergic reaction without rash}

Suppose the patient complains of itching without a rash and no other cause. In that case, it is recommended that symptomatic treatment with antihistamines (diphenhydramine $25-50 \mathrm{mg}$ or cetirizine 5-10 mg before ATD treatment) and skin moisturizer if the skin is dry. Corticosteroid ointment or oral prednisolone (10-20 $\mathrm{mg}$ per day) can be given if there is no improvement. TB treatment continues with close supervision; symptoms usually disappear within a few weeks. $^{20}$

\section{ATD allergic reaction with rash}

If a rash occurs, all ATD must be stopped and the patient must be referred immediately to the referral health facility. In the event of a severe skin reaction involving mucosa or hypotension, give intravenous fluids immediately. ${ }^{21}$

Given the need to complete TB treatment, in referral health facilities efforts can be made to find the type of ATD that causes the allergic reactions by drug challenge:

- After the reaction is under control, ATD is given back gradually one by one, starting with ATD, which is less likely to cause a reaction ( $\mathrm{H}$ or $\mathrm{R}$ ) at low doses, e.g., $50 \mathrm{mg}$ Isoniazid.

- ATD dose increased gradually in 3 days. If no response arises, add one more type of ATD the next day.
- The reaction after giving certain ATD shows that ATD is the cause.

- If the ATD that causes the reaction is known, treatment can be continued without the drug. ${ }^{22,23}$

\section{ATD regimen for allergic reaction with rash}

ATD regimen for allergic reaction with rash is the same as a specific regimen in liver disorders due to ATD, which are:

- If Rifampicin is the cause of allergy, a regimen without Rifampicin is recommended, i.e., two months Isoniazid, Pyrazinamide, and Ethambutol followed by a 10-month follow-up phase of Isoniazid and Ethambutol.

- If isoniazid cannot be used because of an allergy, the regimen is Rifampicin, Pyrazinamide, and Ethambutol for 6-9 months.

- If Pyrazinamide is stopped before the intensive phase is completed due to drug allergy, the total administration of Isoniazid and Rifampicin is extended to 9 months.

- If Isoniazid and Rifampicin cannot be given, streptomycin, ethambutol, and fluoroquinolone are used for 18-24 months. ${ }^{22,23}$

The drugs which cause allergic reactions from the lowest to the highest risk are Isoniazid, Rifampicin, Pyrazinamide, Ethionamide, Cycloserine, Ethambutol, Para-aminosalicylic acid (PAS), and Streptomycin. The desensitization is based on the order of the drug list. ${ }^{24}$ The current guidelines for ATD allergy management are World Health Organization (WHO) guidelines which are described in the Treatment of Tuberculosis Guidelines. If there is rash due to ATD, all drugs must be stopped and re-challenged ATD immediately. ${ }^{23}$ American Thoracic Society/Infectious Disease Society of America (ATS/IDSA) recommends re-challenging ATD can be given 2-3 days after symptoms improve. ${ }^{25}$ 
Table 2. Re-challenging ATD ${ }^{21,24,26}$

\begin{tabular}{|c|c|c|c|c|}
\hline ATD & \multirow{2}{*}{ Rare cause } & Day 1 & Day 2 & Day 3 \\
\hline Isoniazid & & $50 \mathrm{mg}$ & $150 \mathrm{mg}$ & $300 \mathrm{mg}$ \\
\hline Rifampicin & & $75 \mathrm{mg}$ & $300 \mathrm{mg}$ & Full dose \\
\hline Pyrazinamide & & $250 \mathrm{mg}$ & 1 gram & Full dose \\
\hline Ethambutol & The most common cause & $100 \mathrm{mg}$ & $500 \mathrm{mg}$ & Full dose \\
\hline Streptomycin & & $125 \mathrm{mg}$ & $500 \mathrm{mg}$ & Full dose \\
\hline
\end{tabular}

Table 3. Difference between drug challenge and desensitization ${ }^{28}$

\begin{tabular}{lll}
\hline & Drug Challenge & Drug Desensitization \\
\hline Hypersensitivity & Not proven yet & It has been proven \\
Aim & Prove hypersensitivity & Make tolerance to drugs \\
Effects on the immune system & None & Tolerance \\
Risk of an allergic reaction & Yes & Yes \\
Initial dose & $1 / 100-1 / 10$ therapy dose & $1 / 1000000-1 / 100000$ therapy dose \\
Number of steps & $3-5$ & More than 10 \\
Interval & Depending on the reaction of the drug & 15 minutes to 2 hours \\
Action after reaction & Stop the test and manage the patient & Pause the administration and resume when \\
& & symptoms subside according to the protocol \\
\hline
\end{tabular}

The drug challenge starts from the drug with the lowest allergic risk, isoniazid, to the most common one, streptomycin (Table 2). The principle of drug challenging ATD is to find an ATD that causes allergies. $^{21,26}$

The first-line ATD combination is still the best and most effective. ${ }^{27}$ The limited efficacy and many side effects of second-line ATD and the limited use of in vivo and in vitro diagnostics in detecting allergic ATD make desensitization of first-line ATD is the first choice. $^{19,27}$

Desensitization is the act of re-giving allergycausing drugs in small doses and gradually increasing until the initial therapeutic dose is reached. After that, the dose is given according to the standard at regular. ${ }^{10}$ Patients who experience hypersensitivity reactions within 24 hours after drug administration can achieve drug tolerance with rapid oral desensitization between 4 to 20 hours. $^{28}$ Drug challenge does not cause drug tolerance. Desensitization must be distinguished from drug challenges or provocation tests, which are diagnostic tools. Drug challenge aims to find drugs that cause allergies. Small doses used to avoid severe allergic reactions. The difference in drug challenge and desensitization can be seen in Table 3 .

Desensitization induces immune tolerance in allergic reactions that are not immune-mediated and immune-mediated. Desensitization is associated with type I hypersensitivity reactions involving mast cells. Mast cells are activated by antigens that bind to Fc\&RI receptors and $\operatorname{IgE}$ ligands, resulting in the degranulation of mast cells. In vivo and in vitro, a gradual increase in drug dosage will cause mast cells to be hyporeactive against the drug. Desensitized mast cells will not release cytokines IL-6 or TNF, thus there is no inflammatory process. ${ }^{29}$ Desensitization is contraindicated in type II and type III hypersensitivity because interactions between antigens and antibodies can lead to consumption and complement activation. Desensitization in type II and III hypersensitivity cannot cause drug tolerance. ${ }^{29}$

Desensitization in type IV hypersensitivity reactions is rare compared to type I hypersensitivity reactions because of different mechanisms of $\mathrm{T}$ cell activation. ${ }^{29}$ Specific involvement of $\mathrm{T}$ cells can be assessed on positive responses to skin tests and patch tests. ${ }^{29} \mathrm{CD} 4$ or CD8 are dominant cytotoxic functions in type IVc hypersensitivity reactions. Several studies mention that CD25 and CD4 T cells increase during desensitization, and CD8 count decreases from 95\% to $35 \%$. CD4 and CD25 have a suppressive effect on the function of CD8 T lymphocytes. ${ }^{29}$

Hypersensitivity reactions of the two most potent ATD, Rifampicin and isoniazid, are rarely concurrent; if hypersensitivity still continues after the re-challenging process, desensitization should be done. An indication of desensitization is if there are no alternative drugs available or the benefits of drug desensitization outweigh the allergy risks. After the desensitization process is finished, give the drug according to the standard dose. In the process of giving the required dose, it also needs the participation of the pharmaceuticals. ${ }^{5}$ Consider close monitoring of patients with severe hypersensitivity reactions. Patients should receive at 
Table 4. Isoniazid and Rifampicin desensitization protocols 24,31

\begin{tabular}{llll}
\hline Time & Isoniazid (milligram) & Time & Rifampicin (milligram) \\
\hline 07.00 & 0.1 & 07.00 & 0.1 \\
07.15 & 0.5 & 07.15 & 0.5 \\
07.30 & 1 & 07.30 & 1 \\
07.45 & 2 & 07.45 & 2 \\
08.00 & 4 & 08.00 & 4 \\
08.30 & 8 & 08.15 & 8 \\
09.00 & 16 & 08.30 & 16 \\
09.30 & 32 & 08.45 & 32 \\
10.30 & 50 & 09.15 & 50 \\
12.30 & 100 & 10.15 & 75 \\
14.30 & 150 & 12.15 & 100 \\
15.00 & 150 & 16.15 & 150 \\
12.30 & Continue 150 mg every 12 hours & 12.15 & Continue 300 mg every 12 hours \\
\hline
\end{tabular}

least 2 ATDs during desensitization to prevent resistance. ${ }^{21,26}$ There is no consensus on antihistamine or corticosteroid premedication use during the desensitization process, and it should be avoided. ${ }^{5,30}$ In general, isoniazid and Rifampicin desensitization protocols can be seen in Table 4.

ATD desensitization protocol according to the Philadelphia Protocol described in the Guidelines for the Management of Adverse Drug Reaction of Antimycobacterial Agents in general include:

- Start giving ATD according to the first-day dose of drug challenges in Table 3.

- If a reaction occurs after the drug challenge, stop giving the drug and start desensitization from $1 / 100-1 / 1000$ dose the first day after the reaction has subsided.

- Give a doubled dose every hour and until the daily therapeutic dose is reached.

- When the therapeutic dose is reached, continue administering in divided doses for three days, then change to a single dose (e.g., Isoniazid 2x150 mg for three days, then $300 \mathrm{mg}$ for a single dose).

- If during the desensitization process there is an allergy, decrease to the previous dose without allergy and slowly increase it again. ${ }^{24}$

\section{SUMMARY}

The first-line ATD is still the most effective TB drug, but it can have some allergic skin reactions that can affect compliance with TB treatment. Skin allergic reactions due to ATD can range from mild symptoms to severe or life-threatening reactions. The most important things in the management are identifying and stopping the drugs temporarily. Desensitization is one of the management of drug allergy by giving back the drug in small doses and gradually increasing it until the therapeutic dose of the drug does not cause an allergic reaction. Proper management can reduce noncompliance and affect the outcome of TB treatment.

\section{REFERENCES}

1. Jung HY, Park S, Shin B, et al. Prevalence and Clinical Features of Drug Reactions with Eosinophilia and Systemic Symptoms Syndrome Caused by Antituberculosis Drugs: A Retrospective Cohort Study. Allergy, Asthma Immunol Res 2019; 11: 90-103.

2. Dedun AR, Borisagar GB, Solanki RN. Impact of Adverse Drug Reaction of First Line AntiTuberculous Drugs on Treatment Outcome of Tuberculosis under Revised National Tuberculosis Control Programme. Int J Adv Med 2017; 4: 645.

3. Marra F, Marra CA, Bruchet N, et al. Adverse Drug Reactions Associated with First-Line AntiTuberculosis Drug Regimens. Int J Tuberc Lung Dis 2007; 11: 868-875.

4. Farazi A, Sofian M, Jabbariasl M, et al. Adverse Reactions to Antituberculosis Drugs in Iranian Tuberculosis Patients. Tuberc Res Treat 2014; 2014: 1-6.

5. Siripassorn K, Ruxrungtham K, Manosuthi W. Successful Drug Desensitization in Patients with Delayed-Type Allergic Reactions to AntiTuberculosis Drugs. Int J Infect Dis 2018; 68: 6168.

6. R.J. Lehloenya, G. Todd, M. Badri KD. Outcomes of Reintroducing Anti-Tuberculosis Drugs Following Cutaneous Adverse Drug Reactions. Int J Tuberc Lung Dis 2011; 15: 1649-1655.

7. Dua R, Sindhwani G, Rawat J. Exfoliative Dermatitis to All Four First Line Oral AntiTubercular Drugs. Indian J Tuberc 2010; 57: 5356.

8. Medicines Information Center University of Cape Town. Management of Suspected Drug-Induced 
Rash, Kidney Injury and Liver Injury in Adult Patients on TB Treatment and/or Antiretroviral Treatment. 2018.

9. Kobashi Y, Abe T, Shigeto E, et al. Desensitization Therapy for Allergic Reactions to Antituberculous Drugs. Intern Med 2010; 49: 2297-2301.

10. Hamm RL. Drug Allergy: Delayed Cutaneous Hypersensitivity Reactions to Drugs. Cit EMJ Allergy Immunol 2016; 1: 92-101.

11. Weiss ME, Bernstein DI, Blessing-moore $\mathrm{J}$, et al. Drug Allergy: An Updated Practice Parameter. Ann Allergy, Asthma Immunol 2010; 105: 259-273.e78.

12. Ministry of Health Singapore. The National Drug Allergy Reporting Guidelines.

13. Demoly $\mathrm{P}$, Adkinson NF, Bockrow $\mathrm{K}$, et al. International Consensus on Drug Allergy. Allergy Eur J Allergy Clin Immunol 2014; 69: 420-437.

14. Buhari GK, Keren $\mathrm{M}$, Dursun $\mathrm{AB}$, et al. Immediate-Type Hypersensitivity Reactions due to Antituberculosis Drugs: A Successful Readministration Protocol. Ann Allergy, Asthma Immunol 2015; 115: 39-44.

15. Abe R. Immunological Response in StevensJohnson Syndrome and Toxic Epidermal Necrolysis. J Dermatol 2015; 42: 42-48.

16. Unal D, Gelincik A, Elitok A, et al. Impact of High Serum Immunoglobulin E Levels on the Risk of Atherosclerosis in Humans. Asia Pac Allergy 2017; 7: 74.

17. Ohrui T, Zayasu K, Sato E, et al. Pulmonary Tuberculosis and Serum IgE. Clin Exp Immunol 2000; 122: 13-15.

18. Ellertsen LK, Storla DG, Diep LM, et al. Allergic Sensitisation in Tuberculosis Patients at the Time of Diagnosis and Following Chemotherapy. BMC Infect Dis 2009; 9: 1-9.

19. Lehloenya RJ, Dheda K. Cutaneous Adverse Drug Reactions to Anti-Tuberculosis Drugs: State of the Art and into the Future. Expert Rev Anti Infect Ther 2012; 10: 475-486.

20. Piubello, Alberto, Aït-Khaled, Nadia. Caminero, José A. Chiang, Chen-Yuan. Dlodlo, Riitta A. Fujiwara, Paula I. Heldal E. Field Guide for the Management of Drug-Resistant Field Guide for the Management of Drug-Resistant. 2018.
21. Harries, a.; Maher, D.; Graham S. TB/HIV a Clinical Manual. 2004. Epub ahead of print 2004. DOI: 10.1016/S0035-9203(97)90302-3.

22. Kementerian Kesehatan Republik Indonesia. Pedoman Nasional Penanggulangan Tuberkulosis. 2014.

23. World Health Organization. Treatment of Tuberculosis Guidelines. 2010. Epub ahead of print 2010. DOI: 10.1007/s10405-019-0234-x.

24. Flick L, Tuberculosis M, Tuberculosis $\mathrm{P}$, et al. Guidelines for the Management of Adverse Drug Effects of Antimycobacterial Agents.

25. Nahid P, Dorman SE, Alipanah N, et al. Official American Thoracic Society/Centers for Disease Control and Prevention/Infectious Diseases Society of America Clinical Practice Guidelines: Treatment of Drug-Susceptible Tuberculosis. Clin Infect Dis an Off Publ Infect Dis Soc Am 2016; 63: e147e195.

26. Ait-Khaled N, Enarson DA. Tuberculosis A Manual for Medical Students. Who 2003; 148.

27. Thong BY-H, Chia FL-A, Tan S-C, et al. A Retrospective Study on Sequential DesensitizationRechallenge for Antituberculosis Drug Allergy. Asia Pac Allergy 2014; 4: 156.

28. Cernadas JR, Brockow $\mathrm{K}$, Romano A, et al. General Considerations on Rapid Desensitization for Drug Hypersensitivity - A Consensus Statement. Allergy Eur J Allergy Clin Immunol 2010; 65: 1357-1366.

29. Collado-Chagoya R, Hernández-Romero J, EliosaAlvarado GA, et al. Tolerance Induction to Antituberculosis Drugs in a Patient with StevensJohnson Syndrome/Toxic Epidermal Necrolysis Overlap. Allergy Rhinol 2018; 9: 215265671878361.

30. Thong BY-H. Clinical Applications of Drug Desensitization in the Asia-Pacific Region. Asia Pac Allergy 2011; 1: 2.

31. Holland CL, Malasky C, Ogunkoya A, et al. Rapid Oral Desensitization to Isoniazid and Rifampin. Chest 1990; 98: 1518-1519. 